\title{
The Epichloë festucae Antifungal Protein Efe-AfpA Is also a Possible Effector Protein Required for the Interaction of the Fungus with Its Host Grass Festuca rubra subsp. rubra
}

\author{
Ruying Wang ${ }^{1,2} \mathbb{D}$, Simin Luo ${ }^{1,3}$, Bruce B. Clarke ${ }^{1}$ and Faith C. Belanger ${ }^{1, *(\mathbb{D}}$ \\ 1 Department of Plant Biology, Rutgers University, New Brunswick, NJ 08901, USA; \\ ruying.wang@oregonstate.edu (R.W.); 2018120011@njau.edu.cn (S.L.); bruce.clarke@rutgers.edu (B.B.C.) \\ 2 Department of Horticulture, Oregon State University, Corvallis, OR 97331, USA \\ 3 College of Agro-Grassland Science, Nanjing Agricultural University, Nanjing 210095, China \\ * Correspondence: belanger@sebs.rutgers.edu
}

\section{check for}

updates

Citation: Wang, R.; Luo, S.; Clarke, B.B.; Belanger, F.C. The Epichloë festucae Antifungal Protein Efe-AfpA Is also a Possible Effector Protein Required for the Interaction of the Fungus with Its Host Grass Festuca rubra subsp. rubra. Microorganisms 2021, 9, 140. https://doi.org/10.3390/ microorganisms 9010140

Received: 3 December 2020

Accepted: 7 January 2021

Published: 9 January 2021

Publisher's Note: MDPI stays neutral with regard to jurisdictional clai$\mathrm{ms}$ in published maps and institutional affiliations.

Copyright: $\odot 2021$ by the authors. Licensee MDPI, Basel, Switzerland. This article is an open access article distributed under the terms and conditions of the Creative Commons Attribution (CC BY) license (https:// creativecommons.org/licenses/by/ $4.0 /)$.

\begin{abstract}
Strong creeping red fescue (Festuca rubra subsp. rubra) is a commercially important lowmaintenance turfgrass and is often naturally infected with the fungal endophyte Epichloë festucae. Epichloë spp. are endophytes of several cool-season grass species, often conferring insect resistance to the grass hosts due to the production of toxic alkaloids. In addition to insect resistance, a unique feature of the strong creeping red fescue/E. festucae symbiosis is the endophyte-mediated disease resistance to the fungal pathogen Clarireedia jacksonii, the causal agent of dollar spot disease. Such disease resistance is not a general feature of other grass / Epichloë interactions. E. festucae isolates infecting red fescue have an antifungal protein gene Efe-afpA, whereas most other Epichloë spp. do not have a similar gene. The uniqueness of this gene suggests it may, therefore, be a component of the unique disease resistance seen in endophyte-infected red fescue. Here, we report the generation of CRISPR-Cas9 Efe-afpA gene knockouts with the goal of determining if absence of the protein in endophyte-infected Festuca rubra leads to disease susceptibility. However, it was not possible to infect plants with the knockout isolates, although infection was possible with the wild type E. festucae and with complemented isolates. This raises the interesting possibility that, in addition to having antifungal activity, the protein is required for the symbiotic interaction. The antifungal protein is a small secreted protein with high expression in planta relative to its expression in culture, all characteristics consistent with effector proteins. If $E f e-A f p A$ is an effector protein it must be specific to certain interactions, since most Epichloë spp. do not have such a gene in their genomes.
\end{abstract}

Keywords: antifungal protein; endophyte; strong creeping red fescue; symbiosis

\section{Introduction}

Strong creeping red fescue (Festuca rubra subsp. rubra) is a commercially important low-maintenance turfgrass and is often naturally infected with the fungal endophyte Epichloë festucae [1,2]. Epichloë spp. are endophytes of several cool-season grass species, often conferring insect resistance to the grass hosts due to the production of toxic alkaloids [1,2]. For turfgrasses, cultivars containing Epichloë endophytes are desired because of the enhanced insect resistance [3]. In addition to insect resistance, a unique feature of the strong creeping red fescue/E. festucae symbiosis is the endophyte-mediated disease resistance to the fungal pathogen Clarireedia jacksonii (formerly Sclerotinia homoeocarpa) [4], the causal agent of dollar spot disease [5]. Such endophyte-mediated disease resistance is not seen in the cultivated grasses ryegrass (Lolium perenne L.) or tall fescue (Lolium arundinaceum) [4,6]. As discussed previously [6], there is conflicting literature on Epichloë spp. mediated disease resistance in various grass hosts but no field-level resistance has been reported other than for the fine fescues [4].

The mechanism underlying the unique endophyte-mediated disease resistance in strong creeping red fescue is not yet established. We are pursuing the possibility that it may 
be due to the presence of an abundant secreted antifungal protein produced by the fungal endophyte E. festucae. In a previous quantitative transcriptome study of the E. festucaestrong creeping red fescue interaction, the second most abundant fungal transcript was found to encode a small (6278 Daltons) secreted protein similar to antifungal proteins from Penicillium and Aspergillus species [7]. The purified E. festucae antifungal protein, Efe-AfpA, was shown to inhibit the growth of $C$. jacksonii and to cause membrane damage in plate assays [6]. The antifungal protein gene, Efe-afpA (gene model EfM3.0636600, http:/ / csbio-1.csr.uky.edu/endophyte/; GenBank accession MG925781.1) [1] found in E. festucae infecting strong creeping red fescue is not present in most Epichloë genomes for which whole genome sequence is available, being present only in E. baconii, E. aotearoae, and E. inebrians [6,7]. The antifungal activity, transcript abundance and the limited existence of the gene among Epichlö spp. suggested the E. festucae antifungal protein may be a component of the unique endophyte-mediated disease resistance observed in strong creeping red fescue. If so, this protein may have the potential to be utilized as a biological control agent/product for the suppression of dollar spot or other diseases on fine fescues and other important turfgrasses.

A common approach to evaluate the role of a particular protein in a physiological process is to generate a gene knockout, in which the target gene is inactivated, and evaluate the phenotype of the mutant. In Epichlö̈ spp., knockouts of numerous genes have been reported using the approach of homologous recombination to replace the target gene with a selectable marker [8]. More recently, CRISPR-Cas9 technology has emerged as an efficient approach to targeted gene deletions [9]. With this method, single guide RNA (sgRNA) with a 20 bp target sequence directs the Cas 9 endonuclease to precisely generate double strand breaks. Repairing the breaks by nonhomologous end joining is likely to introduce mistakes causing frame shifting, which frequently inactivates the gene of interest. CRISPR-Cas9 technology has been established for animal, plant, yeast, and filamentous fungal systems [10-13]. Here, we report the application of CRISPR-Cas9 gene deletion technology to the E. festucae antifungal protein gene. Two independent gene knockout isolates were obtained but they were unable to infect strong creeping red fescue plants suggesting that Efe-afpA may be essential for infection and association with the host.

\section{Materials and Methods}

\subsection{Construction of Vectors Used for the Knockout and Rescue Transformations}

For vector construction, genomic DNA of the Rose City isolate of E. festucae [14] was extracted from cultures grown on potato dextrose agar or in potato dextrose broth (Difco Laboratories, Detroit, MI, USA) using the method described by Moy et al. [15]. The details of the construction of the vector used for homologous recombination are described in Wang [16].

Two CRISPR-Cas9 vectors were constructed. The vector p-hph-Ptef1-cas9-pksP-gRNA [17], (obtained from the Fungal Genetics Stock Center, Manhattan, KS, USA), which contains the $h p h$ resistance gene, the human codon-optimized cas9 gene with the SV40 nuclear localization sequence, and gRNA targeting the Aspergillus fumigatus polyketide synthase gene $p k s \mathrm{P}$, was modified to have gRNA targeting Efe-afpA. The Efe-afpA gRNA was designed to target the sequence $5^{\prime}$-GGCATTCTGATCACGTATGA-3' immediately upstream of the protospacer-adjacent motif (PAM) sequence AGG in the first exon of Efe-afpA. Primers AFP-Del2-F and AFP-Del2-R (Table S1) were used to change the existing gRNA sequence of p-hph-Ptef1-cas9-pksP-gRNA to the Efe-afpA gRNA target sequence with the Q5 SiteDirected Mutagenesis Kit (New England BioLabs Inc., Ipswich, MA, USA). Since p-hphPtef1-cas9-pksP-gRNA is a large plasmid (>13 kb), PrimeSTAR HS Premix (TaKaRa Bio Inc., Shiga, Japan) was used instead of Q5 Hot Start High-Fidelity 2X Master Mix in the Q5 Site-Directed Mutagenesis Kit. PCR was performed in a $25 \mu \mathrm{L}$ volume with one ng of the vector p-hph-Ptef1-cas9-pksP-gRNA, $0.3 \mu \mathrm{M}$ of each forward and reverse primer (Integrated DNA Technologies, Inc., Coralville, IA, USA) and $12.5 \mu \mathrm{L}$ of PrimeSTAR HS Premix. Two-step PCR was performed by template denaturation at $98^{\circ} \mathrm{C}$ for $10 \mathrm{~s}$ followed 
by 6 min extension at $68^{\circ} \mathrm{C}$ for 30 cycles. The PCR product was used in the remaining steps following the Q5 Site-Directed Mutagenesis Kit manufacturer's protocol. The resulting plasmid was designated pD2P6.

A second CRISPR-Cas9 vector containing a Trichoderma reesei codon-optimized cas 9 with a SV40 nuclear localization sequence was constructed utilizing some of the components of pD2P6 described above and the cas 9 sequence from plasmid pDHt/sk-PC [18], (provided by Gen Zou and Zhihua Zhou). In this construct, the T. reesei codon-optimized cas9 gene includes the T. reesei constitutive pyruvate decarboxylase promoter (Ppdc) and terminator (Tpdc) sequences. The new plasmid was assembled from three fragments. Fragments 1 and 2 were amplified from pD2P6 with primers sets BG2F/FullerGR and FullerGF2/PCseqR1, respectively. The Ppdc-T. reesei codon-optimized cas9-Tpdc fragment from the plasmid $\mathrm{pDHt} / \mathrm{sk}-\mathrm{PC}$ was amplified using primers GTCas9F and GTCas9R to also overlap fragments 1 and 2. The $100 \mu \mathrm{L}$ reactions contained 2X Phusion Green Hot Start II High-Fidelity PCR Master Mix (Thermo Fisher Scientific, Waltham, MA, USA), $0.5 \mu \mathrm{M}$ of each oligonucleotide, and either $125 \mathrm{pg}$ of pD2P6 or $2 \mathrm{ng}$ of $\mathrm{pDHt} / \mathrm{sk}-\mathrm{PC}$ as template. The PCR reaction conditions for fragment 1 and 2 were an initial denaturation step at $98^{\circ} \mathrm{C}$ for $30 \mathrm{~s}$, followed by 30 cycles of $10 \mathrm{~s}$ denaturation at $98^{\circ} \mathrm{C}, 30 \mathrm{~s}$ annealing at $60^{\circ} \mathrm{C}$, and $5 \mathrm{~min}$ extension at $72{ }^{\circ} \mathrm{C}$. The PCR reaction conditions for amplification of the Ppdc-toCas9-Tpdc fragment were an initial denaturation step at $98^{\circ} \mathrm{C}$ for $30 \mathrm{~s}$, followed by 30 cycles of $10 \mathrm{~s}$ denaturation at $98^{\circ} \mathrm{C}, 30 \mathrm{~s}$ annealing at $65^{\circ} \mathrm{C}$, and $3.5 \mathrm{~min}$ extension at $72^{\circ} \mathrm{C}$. PCR products were then purified by using 0.7X Agencourt AMPure XP (Beckman Coulter, Brea, CA, USA) and assembled with Gibson Assembly Master Mix (E2611S; New England BioLabs Inc., Ipswich, MA, USA). In a $20 \mu \mathrm{L}$ reaction, $0.11,0.10$, and 0.06 pmol of each fragment 1,2 , and Ppdc-toCas9-Tpdc, respectively, were mixed with 2X Gibson Assembly Master Mix and incubated at $50^{\circ} \mathrm{C}$ for $1 \mathrm{~h}$. One $\mu \mathrm{L}$ of the assembly reaction was used to transform $25 \mu \mathrm{L}$ NEB 10-beta Electrocompetent E. coli cells (New England BioLabs Inc., Ipswich, MA, USA). The resulting plasmid was designated pG4.

\subsection{Construction of a Plasmid for Complementation of the Efe-afpA Knockouts}

A PCR fragment of the Efe-afpA gene, including $596 \mathrm{bp}$ upstream and $573 \mathrm{bp}$ downstream of the coding sequence, was cloned into the pMiniT 2.0 vector according to the instructions provided with the NEB PCR Cloning Kit (New England Biolabs, Ipswich, MA, USA). The PCR fragment was generated from E. festucae genomic DNA by using primers $60 \mathrm{~F}$ and $60 \mathrm{R}$. The primer sequences include 20 and $21 \mathrm{bp}$ homologous to the $5^{\prime}$ and $3^{\prime}$ upstream and downstream sequences of the Efe-afp $A$ gene, respectively, and $40 \mathrm{bp}$ of tag sequences not found in the $E$. festucae genome. The nonhomologous sequences provided sequences that could be used as primers for screening transformants for successful integration of the intact Efe-afpA gene PCR fragment.

\subsection{Protoplast Preparation}

Protoplast preparation and transformation of the wild type Rose City isolate and the two knockout isolates were modified from the procedures described by Turgeon et al. [19] and Sonderegger et al. [20]. The fungal isolates were grown in $50 \mathrm{~mL}$ potato dextrose broth medium in $200 \mathrm{~mL}$ flasks for two weeks at room temperature with shaking at 80 $\mathrm{rpm}$. The fungal mycelium from two flasks was collected in $50 \mathrm{~mL}$ tubes by centrifugation at $5000 \mathrm{rpm}$ for $10 \mathrm{~min}$ at $4{ }^{\circ} \mathrm{C}$ in a Sorvall Legend X1R centrifuge. The supernatant was discarded and the mycelium was transferred to sterile paper towels and blotted dry. The mycelium was then distributed to four $50 \mathrm{~mL}$ centrifuge tubes in $0.8-1 \mathrm{~g}$ aliquots. A solution of lysing enzymes from Trichoderma harzianum (Item L1412, Sigma-Aldrich, St. Louis, MO, USA) was prepared by dissolving the enzyme powder in $5 \mathrm{~mL}$ lysis buffer (50 $\mathrm{mM}$ phosphate buffer, $\mathrm{pH} 5.8,0.7 \mathrm{M} \mathrm{KCl}$ ). After the powder was dissolved, an additional $10 \mathrm{~mL}$ of lysis buffer was added, and the solution was filter sterilized. The sterilized enzyme solution was then distributed evenly to the four tubes of fungal mycelium and the volume in each tube was brought to $10 \mathrm{~mL}$ with lysis buffer. The samples were incubated 
at $30^{\circ} \mathrm{C}$ with gentle shaking $(50 \mathrm{rpm})$ for $18 \mathrm{~h}$. The protoplasts were separated from intact mycelium and cell wall debris by filtering the suspension through four layers of sterile cheesecloth. The protoplasts were pelleted by centrifugation at $5000 \mathrm{rpm}$ for $5 \mathrm{~min}$ at $4{ }^{\circ} \mathrm{C}$ in a SS-34 fixed angle rotor. The protoplast pellets from the four tubes were resuspended in a total volume of $10 \mathrm{~mL} 0.7 \mathrm{M} \mathrm{KCl}$ and combined into one tube and were centrifuged again as before. The protoplast pellet was then washed three times with $10 \mathrm{~mL}$ STC buffer (1.2 M sorbitol, $10 \mathrm{mM}$ Tris- $\mathrm{HCl}$, pH 7.5, $50 \mathrm{mM} \mathrm{CaCl}_{2} \mathrm{H}_{2} \mathrm{O}$ ), pelleting the cells between washes as before. The final protoplast pellet was resuspended in $100 \mu \mathrm{L}$ STC buffer. The protoplasts were counted using a hemocytometer and adjusted to a density of approximately $10^{8} \mathrm{~mL}^{-1}$ with STC.

\subsection{Fungal Transformation and Screening of Transformants}

All transformations used the polyethylene glycol-mediated protoplast transformation method as described previously [19]. For the unsuccessful CRISPR-Cas9 knockout, $10 \mu \mathrm{g}$ of plasmid pD2P6 in $25 \mu \mathrm{L}$ was used. For the CRISPR-Cas9 knockout, $10 \mu \mathrm{g}$ of in vitro transcribed gRNA and $10 \mu \mathrm{g}$ of pG4 plasmid in a total of $25 \mu \mathrm{L}$ were co-transformed into approximately $6 \times 10^{6}$ E. festucae Rose City protoplasts. Single-guide RNA was generated in vitro using GeneArt Precision gRNA Synthesis Kit (Thermo Fisher Scientific, Waltham, MA, USA) according to the manufacturer's instructions with primers gF2 and gR2.

For complementation, protoplasts of the two knockout isolates were co-transformed with 2-5 $\mu \mathrm{g}$ of plasmid FB009 (Addgene plasmid \#119707, Watertown, MA, USA) containing the selectable marker for the antibiotic G418 and either $2.5 \mu \mathrm{g}$ of a PCR fragment containing the Efe-afpA gene or $5 \mu \mathrm{g}$ of the complementation plasmid described above.

Transformed protoplasts were plated into molten regeneration medium as described by Turgeon et al. [19] and incubated overnight at $30{ }^{\circ} \mathrm{C}$. The plates were then overlaid with $10 \mathrm{~mL}$ of PDA containing $150 \mu \mathrm{g} \mathrm{mL}^{-1}$ hygromycin for the knockouts or $200 \mu \mathrm{g} \mathrm{mL}^{-1} \mathrm{G} 418$ for the rescue transformants. Transformed fungal colonies that emerged through the antibiotic layer were grown on PDA plates containing the appropriate antibiotic overlaid with cellophane.

For screening the transformants fungal genomic DNA was extracted by using the DNeasy Plant Pro Kit (Qiagen, Germantown, MD, USA). Knockout transformants were identified by sequencing PCR products amplified from the Efe-afpA genomic region with primers cafp-f and AFPr. The rescue transformants were identified by sequencing PCR products amplified with the primers COM1F and COM1R. The PCR reaction conditions were one cycle at $98^{\circ} \mathrm{C}$ for $30 \mathrm{~s} ; 30$ cycles at $98^{\circ} \mathrm{C}$ for $10 \mathrm{~s}, 60{ }^{\circ} \mathrm{C}$ for $30 \mathrm{~s}$, and $72{ }^{\circ} \mathrm{C}$ for $30 \mathrm{~s}$; and one cycle at $72{ }^{\circ} \mathrm{C}$ for $5 \mathrm{~min}$. PCR products were sequenced directly. For each sequencing reaction, a $5 \mu \mathrm{L}$ aliquot of each PCR product was treated with $2 \mu \mathrm{L}$ ExoSAP-IT (USB Corp., Cleveland, OH, USA) to remove unincorporated primers and excess dNTPs. The ExoSAP-IT reaction was performed at $37^{\circ} \mathrm{C}$ for $15 \mathrm{~min}$ followed by heating at $80{ }^{\circ} \mathrm{C}$ for $15 \mathrm{~min}$ to inactivate the enzymes, and then sequenced (Genewiz, Inc., South Plainfield, NJ, USA). Single spore isolates of the CRISPR-Cas9 knockout mutants and the rescue isolates were generated by washing a plate of the knockout and rescue isolates with sterile water and plating dilutions of the water containing spores onto new plates. The status of isolated colonies originating from single spores was confirmed by sequencing PCR fragments as described for the primary screening.

\subsection{Strong Creeping Red Fescue Inoculation with E. festucae Isolates}

The knockout and rescue isolates and the wild type Rose City isolate were used to inoculate germinating endophyte-free seedlings of the strong creeping red fescue cultivar Kent using a modification of the method described by Latch and Christensen [21]. Seeds were sterilized as described by Johnson-Cicalese et al. [22] and germinated in the dark on $3 \%(w / v)$ water agar in $10 \mathrm{~cm}$ square petri dishes. The petri dishes were maintained in an upright position to allow the seedlings to grow gravitropically. After five days in the dark, they were put under light for two days. The seed coats were removed aseptically and a 
$10 \mu \mathrm{L}$ aliquot of potato dextrose broth was applied to the crown area of each seedling. A small piece of fungal tissue from the edge of a colony growing on potato dextrose agar was homogenized in $1 \mathrm{~mL}$ sterile water in a mortar and a $5 \mu \mathrm{L}$ aliquot of the homogenate was applied to the crown of each seedling on top of the potato dextrose broth. After about eight days, fungal hyphae could be seen growing around the seedling. At that point, the seedling was wounded in the crown region with a 30-gauge needle (BD Ultra-Fine Insulin Syringes, Franklin Lakes, NJ, USA) to allow the growing fungal hyphae to enter the plant. Three to five days after wounding the seedlings were transferred to Fafard Canadian Grow Mix 2 (Agawam, MA, USA). The transplanted seedlings were acclimated in the lab at room temperature for several weeks and then transferred to the greenhouse. After several new tillers had emerged, the inoculated plants were screened for endophyte infection by an immunoblot assay (Phytoscreen, Agrinostics, Ltd. Co., Watkinsville, GA, USA) and positive plants were confirmed microscopically. For microscopic analysis, leaves or leaf sheaths were pretreated by submerging tissue in $95 \%$ ethanol in 24 -well microtiter plates for more than $4 \mathrm{~h}$ to remove chlorophyll. They were then incubated in aniline blue-lactic acid stain [23] for $15 \mathrm{~min}$ at $40^{\circ} \mathrm{C}$ and examined by microscopy for intercellular fungal hyphae.

Inoculation with the complemented knockout isolates was further confirmed by generating PCR fragments with primers COM1F and RescueR from DNA isolated from the inoculated plants. The PCR fragment was sequenced confirming the fragment was from $E f e-a f p A$. The COM1F sequence is part of the $60 \mathrm{~F}$ primer used to amplify the Efe-afpA gene for complementation of the knockout isolates. This serves as a tag for the complementation sequence since it is not normally present in the E. festucae genome.

\subsection{PacBio Long Read Sequencing}

Genomic DNA of the wild type E. festucae Rose City isolate was isolated as described by Dellaporta et al. [24]. Library preparation and sequencing was done by Genewiz (South Plainfield, NJ, USA). PacBio SMRTbell libraries were prepared per the manufacturer's instructions and sequenced on the PacBio Sequel platform with v3.0 chemistry. The genome sequence of the wild type Rose City isolate was de novo assembled by Genewiz using HGAP4 software with default parameters, generating 46 contigs. This Whole Genome Shotgun project has been deposited at DDBJ/ENA/GenBank under the accession JADWOS000000000. The version described in this paper is version JADWOS010000000. The BioProject ID is PRJNA677971.

\subsection{Quantitative RT-PCR}

Total RNA was isolated from frozen mycelium of the E. festucae Rose City isolate and from leaf sheath tissue of the Rose City isolate-infected plant tissue. The tissues were homogenized in a HT Mini bead beater (Ops Diagnostics, Lebanon, NJ, USA) and RNA extracted using the ZR Fungal/Bacterial RNA MiniPrep Kit (Zymo Research, Irvine, CA, USA) according to the manufacturer's instructions. A TURBO DNA-free kit (Invitrogen, Carlsbad, CA, USA) was used to remove any genomic DNA in the RNA samples. cDNA synthesis was performed on $1.5 \mu \mathrm{g}$ RNA using the High-Capacity cDNA Reverse Transcription Kit (Applied Biosystems, Foster City, CA, USA). Quantitative RT-PCR was performed using the Power SYBR Green PCR Master Mix (Applied Biosystems) on a StepOnePlus RT-PCR system (Applied Biosystems) with three technical replicates per sample. Efe-afpA transcripts were normalized to the expression levels of elongation factor 2 (EfM3.021210) and 40S ribosomal protein S22 (EfM3.016650) using primers described by Chujo and Scott [25] and the $2^{-\Delta \Delta C t}$ method as described by Livak and Schmittgen [26]. Primer sequences used for quantitative RT-PCR are given in Table S1. 


\section{Results}

3.1. The Homologous Recombination Approach Was Unsuccessful In Generating a Gene Deletion of the E. festucae Antifungal Protein Gene

The first attempt at generating a knockout of the antifungal protein gene used the well-established homologous recombination method, which has been successful for many Epichloë genes [8]. In this method, a plasmid for transformation is constructed in which sequences identical to the region of the targeted gene flank an antibiotic resistance gene. When such a plasmid is transformed into protoplasts of the fungus, homologous recombination occurs at the sites of the flanking sequences and the antibiotic resistance gene replaces the targeted gene generating the gene knockout. Filamentous fungi typically require long flanking sequences for efficient homologous recombination to occur. Generally flanking sequences of 1.5 to $2.5 \mathrm{~kb}$ are used and the frequencies of gene knockouts range from $1-25 \%$ [8].

The transformation vector for the homologous recombination method was made by ligating the hygromycin phosphotransferase $(h p h)$ cassette to $598 \mathrm{bp}$ left flank and $634 \mathrm{bp}$ right flank of the antifungal protein gene amplified from E. festucae genomic DNA. The antifungal protein gene is a small gene surrounded by A/T rich repeated sequences, which made it impossible to use longer unique flanking sequences for this approach. Over 250 hygromycin resistant transformants from the homologous recombination method were screened by PCR but none were the desired gene replacement mutant. Although, the homologous recombination approach is widely used in Epichloë species, this conventional fungal gene knockout method was unsuccessful in our study. These results are likely because only short unique upstream and downstream regions were available for vector construction since the antifungal protein gene is flanked on both sides by repeated sequences.

\subsection{Efe-afpA Knockouts Recovered by Using CRISPR-Cas9}

The CRISPR-Cas9 technology has recently become the method of choice for gene knockouts in many organisms because it requires only 20 bases of sequence identity to the target gene [27]. The first attempt at generating an Efe-afpA gene knockout using the CRISPR-Cas9 approach used a construct that was successfully used in Aspergillus fumigatus and which had a Cas9 enzyme sequence that was codon-optimized for use in human cells [17]. However, that construct was not successful in generating a knockout in E. festucae.

Liu et al. [18] reported that the human codon-optimized cas9 gene did not function in Trichoderma reesei but that a construct with a Trichoderma codon-optimized cas 9 gene did. Phylogenetically, Epichloë is more closely related to Trichoderma than it is to Aspergillus. Both Epichloë and Trichoderma are genera in the order Hypocreales within the class Sordariomycetes, whereas Aspergillus is in the order Eurotiales within the class Eurotiomycetes. We, therefore, modified the first CRISPR-Cas9 construct to incorporate the T. reesei codonoptimized cas 9 gene rather than the human codon-optimized gene. The modified construct was successful in generating Efe-afpA gene knockouts.

To knock out Efe-afpA the sgRNA was designed to target a sequence in the first exon of the gene. The Cas 9 endonuclease generated double-strand breaks at the target site of the antifungal protein gene, and mutations were introduced when repairing the break. After screening 42 hygromycin resistant isolates, two Efe-afp $A$ knockout isolates were identified. One, 1a-7t $8 \mathrm{~s} 3$, had a single $\mathrm{T}$ insertion at the Cas 9 target site, which immediately introduced an early stop codon (Figure 1). The second knockout isolate, 1c-3s5, had a 10 bp deletion of the Efe-afpA gene coupled with an insertion of $1625 \mathrm{bp}$ at the target site, which was identified as a fragment from the transformation vector (Figure 1 and Supplemental Figure S1).

The two knockout isolates were complemented by transformation with the intact Efe-afp $A$ gene including $596 \mathrm{bp} 5^{\prime}$ of the coding sequence and $573 \mathrm{bp} 3^{\prime}$ of the coding sequence. Two independent complemented isolates of each $\triangle E f e-a f p A$ isolate were recovered, designated 1a-7t8s3-B, 1a-7t8s3-I, 1c-3s5-X, and 1c-3s5-L. 


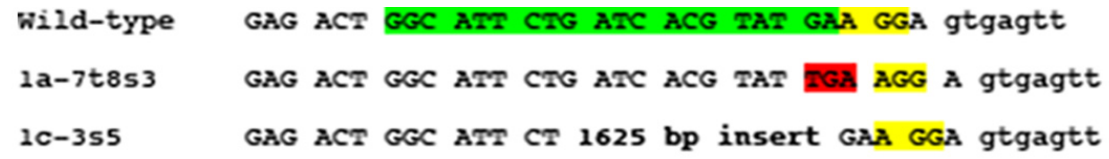

Figure 1. Sequence alignment of part of the first exon of Efe-afpA of the wild-type Rose City isolate of E. festucae, and the two knockout mutants. A single base pair insertion (T) in 1a-7t8s 3 introduced a stop codon, highlighted in red. Knockout isolate 1c-3s5 had a 10 bp deletion of Efe-afpA and a $1625 \mathrm{bp}$ insertion, which was a fragment from the transformation vector pG4. The target guide RNA sequence and PAM sequence designed for CRISPR-Cas9 are highlighted in green and yellow, respectively. Upper case letters indicate $E f e$-afpA coding sequence and lower case letters indicate intron sequence.

Both of the knockout isolates exhibited slightly slower growth rates on potato dextrose agar than the wild type Rose City isolate, which was not fully restored in the complemented isolates (Figure 2). Microscopic observation of the hyphae of the knockout and complemented isolates did not reveal any differences with the wild type.

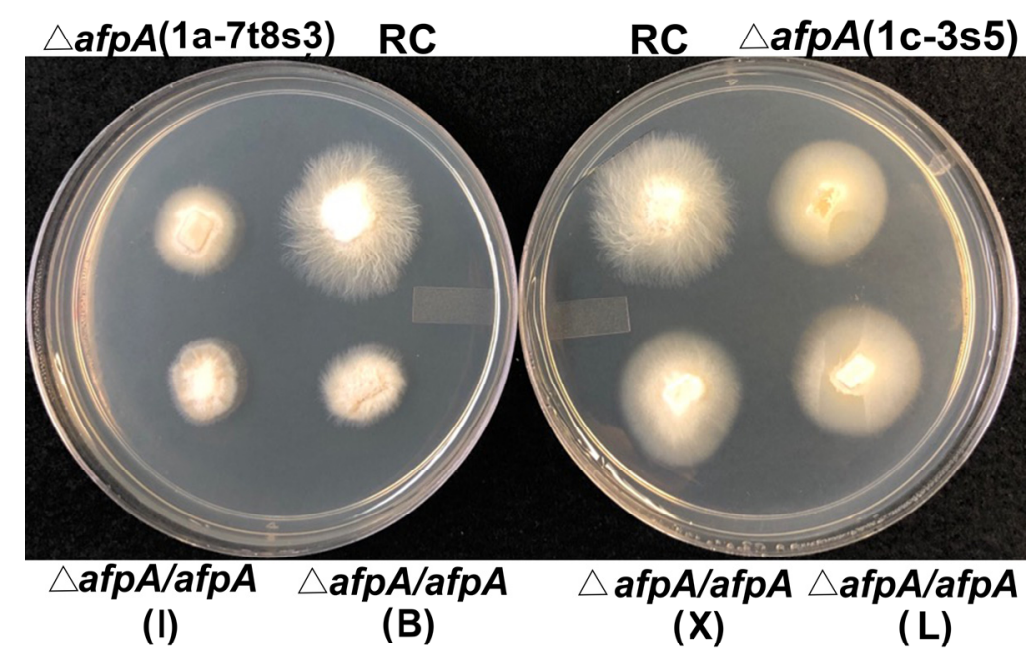

Figure 2. Comparison of colony morphology of the wild type E. festucae Rose City (RC) isolate, the two knockout isolates, and the four independent complemented knockout isolates on potato dextrose agar plates. The letter designations of the four complemented knockout isolates (I, B, X, and L) are given in parentheses.

\subsection{Efe-afpA Knockout Isolates Were Unable to Infect Strong Creeping Red Fescue}

The reason for generating the Efe-afpA knockouts was to determine the effect of the fungus lacking Efe-afpA on the dollar spot susceptibility of strong creeping red fescue by inoculating uninfected plants with the knockout isolates and comparing disease levels with the wild type endophyte-infected plants. No successful inoculations of the knockout isolates were obtained, precluding such a comparison. The inoculation method used was successful in inoculating strong creeping red fescue seedlings with the wild type Rose City isolate of E. festucae, as well as the complemented knockout isolates (Table 1). However, the knockout isolates appeared unable to infect strong creeping red fescue seedlings. The $\triangle E f e$-afp $A$ knockout isolates apparently have reduced ability to infect their host grass. That neither of the two independent $\triangle E f e-a f p A$ isolates was able to infect the strong creeping red fescue seedlings and that the wild type and three of the four independent complemented isolates were able to infect suggests that Efe-AfpA may have a role in the ability of the fungus to infect and establish a symbiotic association with the plant. 
Table 1. Results of inoculating strong creeping red fescue (Festuca rubra subsp. rubra) seedlings with the Epichloë festucae wild type Rose City (RC) isolate, the two $\triangle E f e-a f p A$ knockout isolates, and the four $\triangle E f e-a f p A / E f e-a f p A$ complemented isolates.

\begin{tabular}{ccc}
\hline & Not Infected & Infected \\
\hline Wild type RC & 97 & 8 \\
$\Delta E f e-a f p A(1 \mathrm{a}-7 \mathrm{t} 8 \mathrm{~s} 3)$ & 180 & 0 \\
$\Delta E f e-a f p A(1 \mathrm{c}-3 \mathrm{~s} 5)$ & 180 & 0 \\
$\Delta E f e-a f p A / E f e-a f p A(1 \mathrm{a}-7 \mathrm{t} 8 \mathrm{~s} 3-\mathrm{B})$ & 74 & 0 \\
$\Delta E f e-a f p A / E f e-a f p A(1 \mathrm{a}-7 \mathrm{t} 8 \mathrm{~s} 3-\mathrm{I})$ & 64 & 1 \\
$\Delta E f e-a f p A / E f e-a f p A(1 \mathrm{c}-3 \mathrm{~s} 5-\mathrm{X})$ & 50 & 2 \\
$\Delta E f e-a f p A / E f e-a f p A(1 \mathrm{c}-3 \mathrm{~s} 5-\mathrm{L})$ & 54 & 2 \\
\hline
\end{tabular}

\subsection{Comparison of Expression Levels of Efe-afpA In Culture and In Planta}

The inoculation data described above indicated that Efe-afp $A$ may be required for the symbiotic association with the host, suggesting that Efe-Afp $A$ may function as an effector protein (discussed more below). One general characteristic of fungal effector proteins is that they are more highly expressed in association with their hosts than in culture [28]. Quantitative RT-PCR revealed that the expression level of Efe-afpA is over 700-fold higher in planta than in culture when compared with two reference genes (Figure 3).

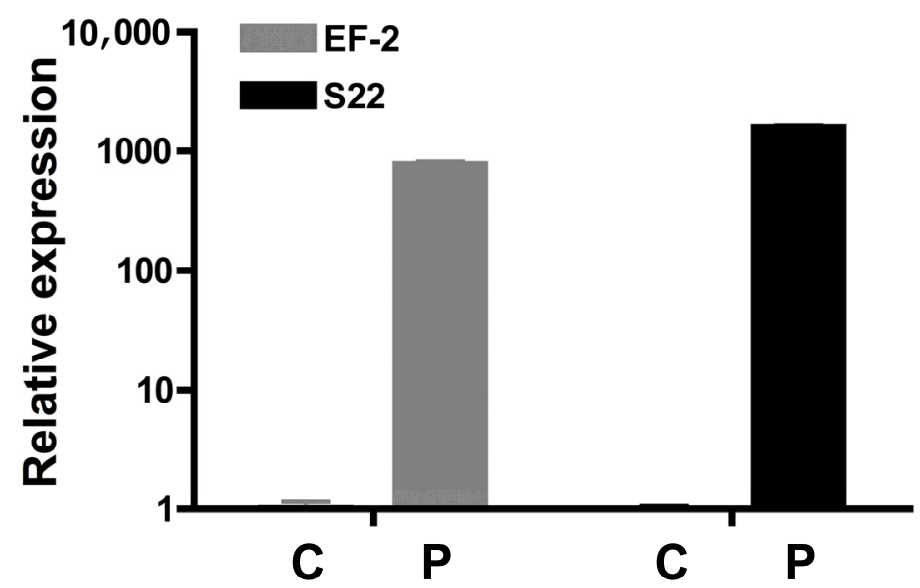

Figure 3. Comparison of expression level of Efe-afpA in culture $(\mathrm{C})$ with expression in planta $(\mathrm{P})$ relative to expression levels of two reference genes, elongation factor 2 (EF-2; gene model EfM3.021210) and 40 S ribosomal protein S22 (S22; gene model EfM3.016650). The y-axis is a logarithmic scale.

\subsection{Identification of Genomic Region of Efe-afpA in the E. festucae Rose City Isolate}

As previously reported, Efe-afpA is the single gene on a $21.4 \mathrm{~kb}$ genome sequence contig from the E. festucae 2368 isolate and is flanked by repeated sequences [1,6, http: / / csbio-1.csr.uky.edu/endophyte/]. To learn more of the genomic context of Efe-afpA, we generated a PacBio long read sequence for the wild type Rose City E. festucae isolate. The characteristics of the sequencing are summarized in Table 2. The sequences were de novo assembled and resulted in 46 contigs, 19 of which could be aligned to the 7 chromosomes of the finished genome sequence of the E. festucae Fl1 isolate, an endophyte of Festuca trachyphylla (hard fescue) [29]. The remaining 27 contigs were matches to the E. festucae Fl1 isolate mitochondrion sequence. Figure 4 depicts the correspondence of the E. festucae Rose City isolate contigs with the 7 chromosomes of the E. festucae Fl1 isolate. As expected for these two E. festucae isolates, there is overall correspondence of the chromosomes, but there is also evidence of apparent chromosomal rearrangements as well as a $400 \mathrm{~kb}$ insertion in the Rose City isolate relative to the Fl1 isolate chromosome 5. 
Table 2. Epichlö̈ festucae Rose City isolate long-read genome assemble.

\begin{tabular}{cc}
\hline Total Contigs & 46 \\
\hline Total bases & $37,116,035$ \\
Max contig length & $4,765,072$ \\
N50 length & $2,773,382$ \\
\hline
\end{tabular}
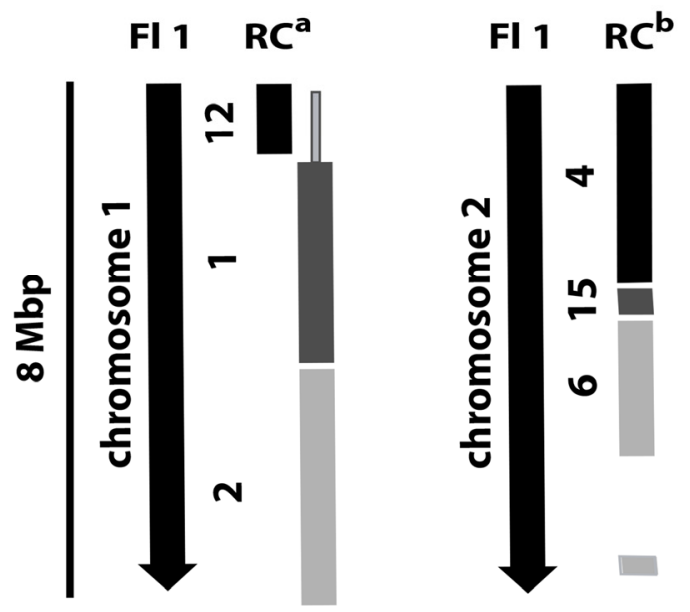

FI 1 RC

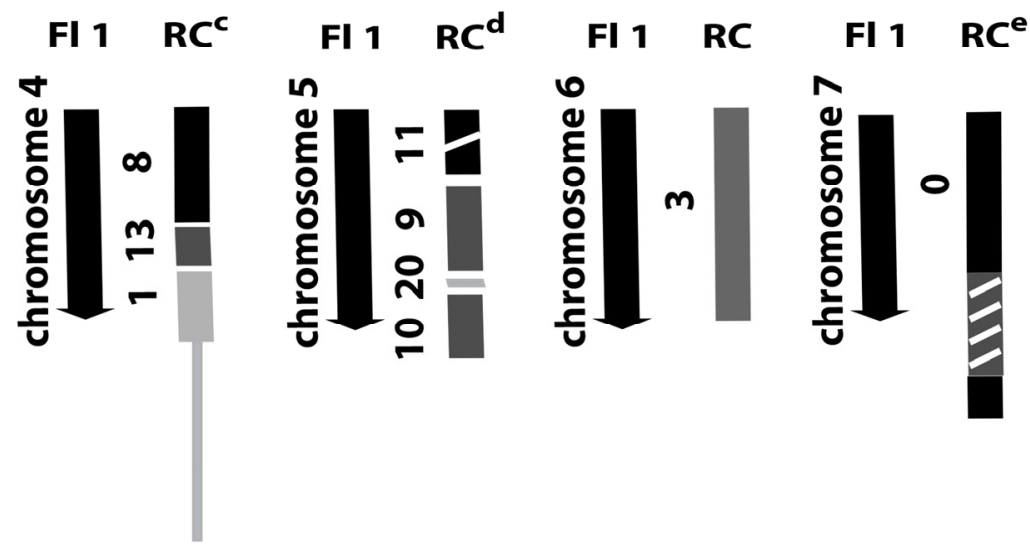

Figure 4. Diagram of relationships of the E. festucae Rose City (RC) isolate genome sequence contigs to the chromosomes of the E. festucae Fl1 isolate. ${ }^{a}$ Line extending from RC contig 1 indicates region of contig 1 that is homologous to Fl1 chromosome $4 .{ }^{\mathrm{b}}$ Gap in RC contig 6 indicates the corresponding region of Fl1 that is found in RC contig $0 .{ }^{\mathrm{c}}$ Line extending from RC contig 1 indicates region of contig 1 that is homologous to Fl1 chromosome 1. ${ }^{\mathrm{d}}$ Break in RC contig 11 indicates an approximately 400,000 bp insert relative to Fl1 chromosome 5. ${ }^{\text {e }}$ Hashed box in RC contig 0 indicates region of contig homologous to Fl1 chromosome 2.

Efe-afp $A$ was localized on the Rose City isolate contig 02, which corresponded to part of chromosome 1 of the Fl1 isolate. The Efe-afaA gene in the Rose City isolate is located in a relative position near the end of chromosome 1 of the Fl1 isolate (Figure 5). Genes surrounding Efe-afp $A$ in the Rose City isolate were syntenous with those genes in the Fl1 isolate, which lacks a gene homologous to Efe-afpA. As shown in the comparison of the $E f e-a f p A$ region of the two E. festucae isolates (Figure 6), the genes surrounding Efe-afpA are conserved but the length of the AT-rich regions between the genes varied between the two isolates and the direction of the coding sequences of EfM3.071850 is opposite between the two isolates. 
A

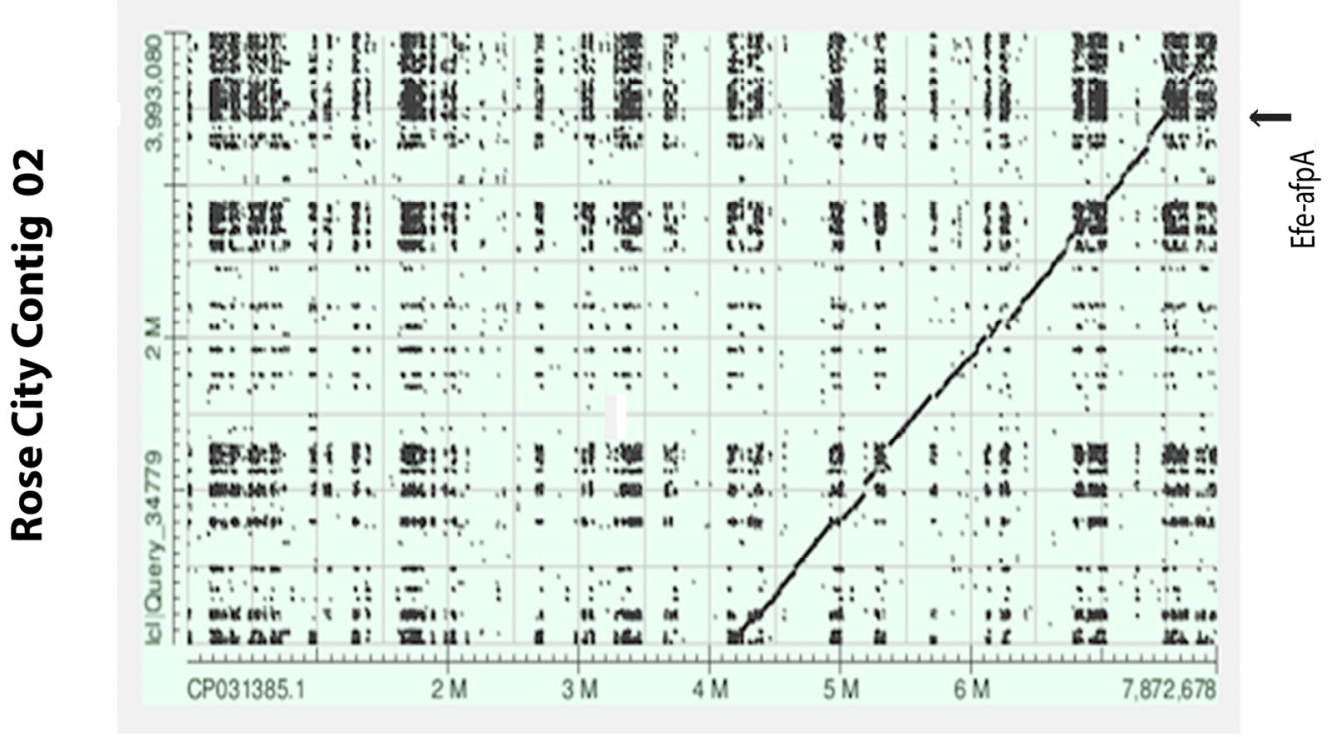

B

\section{Fl 1 Chromosome 1}

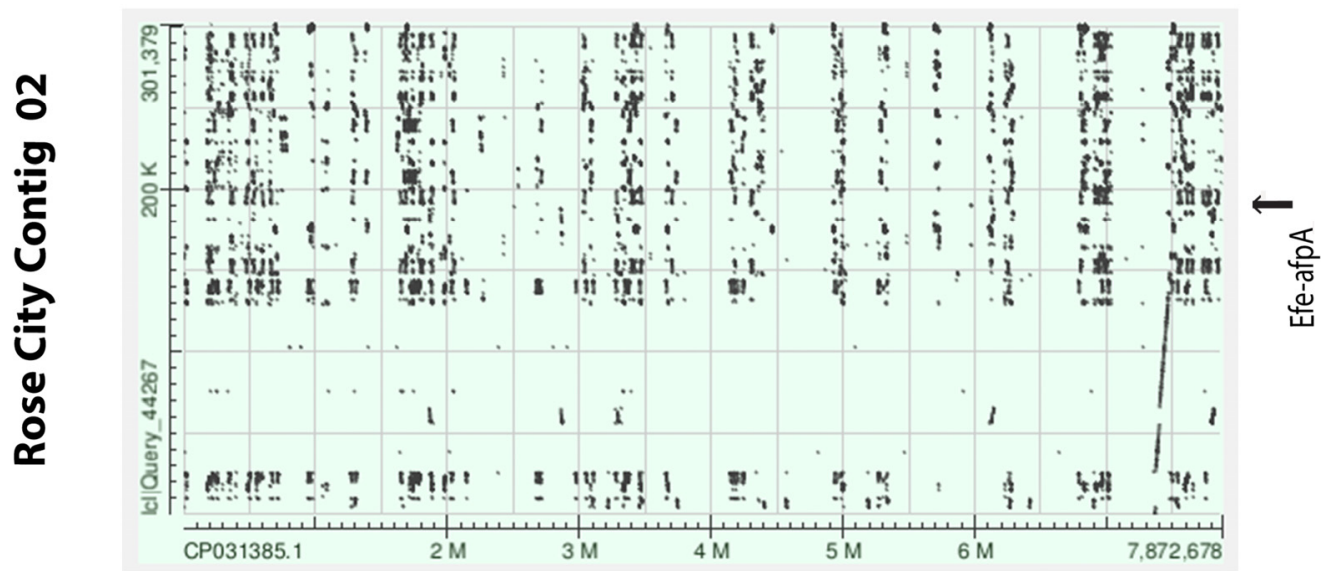

FI 1 Chromosome 1

Figure 5. (A). Screen shot of dot plot comparison of the E. festucae Rose City isolate PacBio contig 02 sequence with the Fl1 chromosome 1 sequence. (B). Screenshot of the dot plot region of Rose City contig 02 sequence that contains Efe-afpA with the Fl1 chromosome 1 sequence. The arrows indicate the position of Efe-afpA in the Rose City sequence.

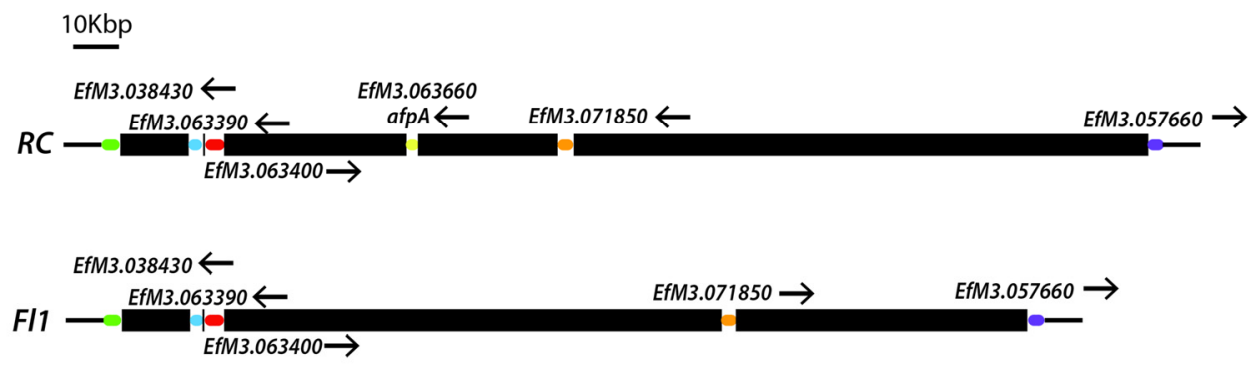

Figure 6. Diagrams from the E. festucae Rose City (RC) isolate contig 2 region containing Efe-afpA and the corresponding region from E. festucae Fl1 isolate chromosome 1 (GenBank accession CP031385.1). Corresponding genes from the two isolates are indicated with the same colors and arrows indicate the directions of the coding sequences. The black bars indicate the AT-rich repeated sequences between genes. 


\section{Discussion}

Here, we successfully applied CRISPR-Cas9 technology to the non-model fungus E. festucae. The CRISPR-Cas9 approach was successful in generating knockouts of a gene that was not feasible using the conventional method of homologous recombination. This was likely due to lack of long enough unique flanking regions required for homologous recombination.

The CRISPR-Cas9 gene editing approach that has been developed for eukaryotic systems requires expression of a functional cas 9 endonuclease gene and of a sgRNA, which targets the desired site of editing. The cas 9 gene was originally identified from the bacterium Streptococcus pyogenes [30] and was codon-optimized for expression in human cells [9]. For use in eukaryotes a nuclear localization signal is required to direct the Cas9 protein to the nucleus. The 7-amino acid SV40 large T antigen nuclear localization signal [31] is often used, appended to the C-terminus of the cas9 coding sequence. The human codon-optimized cas9 gene has been successfully used in several fungal species, including Saccharomyces cerevisiae [32], Neurospora crassa [33], Aspergillus fumigatus [17,34], and Penicillium chrysogenum [35]. However, the same construct that was successfully used in A. fumigatus [17] was not successful in E. festucae. The human codon-optimized cas9 gene was reported to not be effective in Trichoderma reesei [18]. The construct that was successful in generating the Efe-afpA knockouts included a Trichoderma reesei codon-optimized cas 9 sequence with $T$. reesei pyruvate decarboxylase promoter and terminator sequences.

In addition to a codon optimized cas 9 gene, another key component for generating gene knockouts is sgRNA, whose expression is generally driven by a polymerase III promoter. In the unsuccessful construct, the sgRNA was part of the cas 9 containing plasmid and its expression was driven by the $S$. cerevisiae snRNA52 promoter, a RNA polymerase III promoter. The snRNA52 promoter was successful in generating the polyketide synthase gene $p k s P$ sgRNA in A. fumigatus [17]. However, the construct containing the human codon-optimized cas9 gene and the snRNA52 promoter for sgRNA expression was not successful in E. festucae. Another commonly used promoter for sgRNA is the endogenous U6 promoter. However, the U6 promoter in many fungi has not been identified because the fungal U6 gene often has multiple introns [36]. The U6 promoter of Epichloë species has not been identified. Common fungal RNA polymerase II promoters such as $\operatorname{trpC}$ [37] and $g p d A$ [38] have been used in fungal systems to drive expression of sgRNA. However, Arazoe et al. [37] showed significantly less transformation efficiency with the $\operatorname{trp} C$ promoter than with the endogenous U6 promoter. A simpler alternative to in vivo expression of sgRNA is co-transformation of a plasmid with the cas 9 gene with in vitro transcribed gRNAs as used by Liu et al. [18] in T. reesei. This approach was used successfully here in E. festucae.

The CRISPR-Cas9 approach to gene knockouts generally results in small indels in the gene of interest, which typically produce a change in reading frame and an early termination codon. One of the Efe-afpA knockouts recovered here was of this type with a single base pair insert. The other knockout had a $10 \mathrm{bp}$ deletion of Efe-afpA and a $1625 \mathrm{bp}$ insertion at the target site that originated from the transforming plasmid. Similar vector insertions at the target site were reported in A. fumigatus [17], Nodulisporium sp. [39], and Sclerotinia sclerotiorum [40].

The Efe-afp $A$ knockouts were generated with the aim of inoculating them into strong creeping red fescue in order to determine if absence of the antifungal protein gene in endophyte-infected plants leads to disease susceptibility. However, the Efe-afpA knockout isolates could not be inoculated into strong creeping red fescue seedlings. In contrast, the wild type Rose City isolate and the complemented knockout isolates were successfully inoculated into strong creeping red fescue seedlings. Similar evidence has been used to identify some other E. festucae genes as required for the symbiotic interaction. Knockouts of two histone methyltransferases, EfM3.042710 and EfM3.062280, were unable to infect the host plant perennial ryegrass but the complemented knockout isolates were able to infect [41]. The authors proposed that the activity of the histone methyltransferases controlled the expression of genes encoding infection factors. In this study, the inability of 
the knockout isolates to infect the host grass precluded an evaluation of the lack of Efe-afpA on disease resistance or susceptibility of the host.

These results presented here suggest Efe-afpA may have a role in the symbiosis, in addition to having antifungal activity. The interaction of fungal plant pathogens and symbionts with their hosts involves effector proteins, characterized as small-secreted proteins that can be important for colonization or for evasion of host defenses [42-44]. In general, fungal effector proteins interact with a host plant protein either in the apoplastic space or inside the plant cell and interfere with a host plant function [45]. E. festucae expresses numerous small-secreted proteins that may function as effectors in its interaction with the host grass [7]. However, none of these small-secreted proteins have been functionally confirmed as effectors. Hassing et al. [28] analyzed the E. festucae genome sequence for potential effectors and identified 141 candidate genes, one of which was Efe-afpA. The E. festucae antifungal protein has the characteristics of an effector protein in that it is a cysteine-rich small secreted protein and its expression is considerably higher in the infected plant tissue than in culture $[28,46]$. In addition to the quantitative PCR data presented here, Efe-afp $A$ was among the most abundant fungal transcripts in transcriptome studies of E. festucae infected leaf sheath and inflorescence tissues [7,47]. The inoculation data presented here suggests that $E f e$-AfpA may be an effector protein required for the symbiotic interaction of E. festucae and its host grass strong creeping red fescue. However, if Efe-AfpA is an effector protein it must be specific to certain interactions, since most Epichloë spp. do not have such a gene in their genomes [6].

In addition to its antifungal activity and the potential that it may be an effector protein, $E f e-A f p A$ is interesting from an evolutionary point of view, since most Epichloë spp. do not have a similar gene. Is the distribution of Efe-afpA among Epichloë spp. due to gene loss or gene gain? Phylogenetic analysis of nonhybrid Epichloë spp. did not reveal a clear lineage that could be proposed as a point of gene gain or gene loss [6]. The antifungal protein gene in the basal lineage, E. inebrians, was more similar to a gene in Pochonia chlamydosporia, a nematode egg parasitic fungus, than to the antifungal protein genes in the more derived lineages of E. festucae, E. baconii, and E. aotearoae suggesting horizontal gene transfer from Po. chlamydosporia to E. inebrians [6]. Additional research will be required to determine the origin of the patchy distribution of antifungal protein genes among Epichloë spp.

Analysis of the E. festucae Fl1 genome revealed the genome is structured such that transposon rich AT repeat blocks largely lacking genes are interspersed with gene-rich regions that are largely repeat free [29]. The authors proposed that genes within such fast-evolving AT-rich blocks may contribute to strain adaptation leading to such regions hosting lineage-specific genes. Efe-afpA is a single gene located within an AT-rich block and is lineage specific. The long read sequence data generated here to determine the genomic context of Efe-afpA will also be a resource for future studies on genome evolution among E. festucae isolates.

In summary, here we have demonstrated the utility of the CRISPR-Cas9 approach to gene deletion in Epichloë. For Efe-afpA, this approach was critical since the standard approach of homologous recombination was not possible due to the lack of long $5^{\prime}$ and $3^{\prime}$ unique regions required for recombination. Unexpectedly, both of the independent $\Delta E f e-$ afp $A$ isolates were unable to infect host plant seedlings, although 3 of the 4 complemented knockout isolates were able to infect. Based on these results our hypothesis is that in addition to having antifungal activity, Efe-AfpA may be an effector protein required for infection and the symbiosis of $E$. festucae with its host grass strong creeping red fescue. Additional research will be required to determine if $E f e-A f p A$ is indeed an effector protein.

Supplementary Materials: The following are available online at https:/ / www.mdpi.com/2076-260 7/9/1/140/s1, Figure S1: Sequence alignment of mutated Efe-afpA region of wild type isolate Rose City and $\triangle E f e-a f p A$ knockout isolate 1c-3s5, Table S1: Sequences of oligonucleotide primers used in this study. 
Author Contributions: Conceptualization, R.W., S.L., B.B.C., and F.C.B. methodology, R.W., S.L., B.B.C., and F.C.B.; data curation, F.C.B.; writing-original draft preparation, R.W.; writing-review and editing, R.W., S.L., B.B.C., and F.C.B.; visualization, R.W.; S.L.; supervision, F.C.B.; project administration, F.C.B.; funding acquisition, B.B.C. and F.C.B. All authors have read and agreed to the published version of the manuscript.

Funding: This research was funded by the United States Golf Association, the Rutgers Center for Turfgrass Science, and the USDA National Institute of Food and Agriculture Hatch project accession number 1007068 through the New Jersey Agricultural Experiment Station, Hatch project NJ12140.

Data Availability Statement: The data presented in this study are openly available at DDBJ/ENA/GenBank under the accession JADWOS000000000.

Acknowledgments: We thank Gen Zou and Zhihua Zhou for the plasmid pDHt/sk-PC. Plasmid p-hph-Ptef1-cas9-pksP-gRNA was obtained from the Fungal Genetics Stock Center, Manhattan, Kansas. We thank William Meyer for the endophyte-free strong creeping red fescue seeds.

Conflicts of Interest: The authors declare no conflict of interest. The funders had no role in the design of the study; in the collection, analyses, or interpretation of data; in the writing of the manuscript, or in the decision to publish the results.

\section{References}

1. Schardl, C.L.; Young, C.A.; Hesse, U.; Amyotte, S.G.; Andreeva, K.; Calie, P.J.; Fleetwood, D.J.; Haws, D.C.; Moore, N.; Oeser, B.; et al. Plant-symbiotic fungi as chemical engineers: Multi-genome analysis of the Clavicipitaceae reveals dynamics of alkaloid loci. PLoS Genet. 2013, 9, e1003323. [CrossRef]

2. Tadych, M.; Bergen, M.S.; White, J.F., Jr. Epichloë spp. associated with grasses: New insights on life cycles, dissemination and evolution. Mycologia 2014, 106, 181-201. [CrossRef] [PubMed]

3. Funk, C.R.; White, R.H.; Breen, J.P. Importance of Acremonium endophytes in turfgrass breeding and management. Agric. Ecosyst. Environ. 1993, 44, 215-232. [CrossRef]

4. Clarke, B.B.; White, J.F., Jr.; Hurley, H.R.; Torres, M.S.; Sun, S. Endophyte-mediated suppression of dollar spot disease in fine fescues. Plant Dis. 2006, 90, 994-998. [CrossRef] [PubMed]

5. Salgado-Salazar, C.; Beirn, L.A.; Ismaiel, A.; Boehm, M.J.; Carbone, I.; Putman, A.I.; Tredway, L.P.; Clarke, B.B.; Crouch, J.A. Clarireedia: A new fungal genus comprising four pathogenic species responsible for dollar spot disease of turfgrass. Fungal Biol. 2018, 122, 761-773. [CrossRef]

6. Tian, Z.; Wang, R.; Ambrose, K.V.; Clarke, B.B.; Belanger, F.C. The Epichloë festucae antifungal protein has activity against the plant pathogen Sclerotinia homoeocarpa, the causal agent of dollar spot disease. Sci. Rep. 2017, 7, 5643. [CrossRef]

7. Ambrose, K.V.; Belanger, F.C. SOLiD-SAGE of endophyte-infected red fescue reveals numerous effects on host transcriptome and an abundance of highly expressed fungal secreted proteins. PLoS ONE 2012, 7, e53214. [CrossRef]

8. Scott, B.; Takemoto, D.; Tanaka, A.; Young, C.; Bryant, M.; May, K. Functional analysis of the Epichloë festucae-perennial ryegrass symbiosis. In Proceedings of the 6th International Symposium on Fungal Endophytes of Grasses, Christchurch, New Zealand, 25-28 March 2007; pp. 433-441.

9. Jinek, M.; East, A.; Cheng, A.; Lin, S.; Ma, E.; Doudna, J. RNA-programmed genome editing in human cells. eLife 2013, 2, e00471. [CrossRef]

10. Wang, H.; LaRussa, M.; Qi, L.S. CRISPR/Cas9 in genome editing and beyond. Annu. Rev. Biochem. 2016, 85, 227-264. [CrossRef]

11. Zhang, Y.; Malzahn, A.A.; Sretenovic, S.; Qi, Y. The emerging and uncultivated potential of CRISPR technology in plant science. Nat. Plants 2019, 5, 778-794. [CrossRef]

12. Cai, P.; Gao, J.; Zhou, Y. CRISPR-mediated genome editing in non-conventional yeasts for biotechnological applications. Microb. Cell Fact. 2019, 18, 63-75. [CrossRef] [PubMed]

13. Schuster, M.; Kahmann, R. CRISPR-Cas9 genome editing approaches in filamentous fungi and oomycetes. Fungal Genet. Biol. 2019, 130, 43-53. [CrossRef] [PubMed]

14. Tredway, L.P.; White, J.F.; Gaut, B.S.; Reddy, P.V.; Richardson, M.D.; Clarke, B.B. Phylogenetic relationships within and between Epichloë and Neotyphodium endophytes as estimated by AFLP markers and rDNA sequences. Mycol. Res. 1999, 103, 1593-1603. [CrossRef]

15. Moy, M.; Li, H.M.; Sullivan, R.; White, J.F., Jr.; Belanger, F.C. Endophytic fungal $\beta$-1,6-glucanase expression in the infected host grass. Plant Physiol. 2002, 130, 1298-1308. [CrossRef] [PubMed]

16. Wang, R. Investigation of Epichloë festucae—Strong Creeping Red Fescue Mutualistic and Antagonistic Interaction. Ph.D. Thesis, Rutgers University, New Brunswick, NJ, USA, 2019.

17. Fuller, K.K.; Chen, S.; Loros, J.J.; Dunlap, J.C. Development of the CRISPR/Cas9 system for targeted gene disruption in Aspergillus fumigatus. Eukaryot. Cell 2015, 14, 1073-1080. [CrossRef] [PubMed]

18. Liu, R.; Chen, L.; Jiang, Y.; Zhou, Z.; Zou, G. Efficient genome editing in filamentous fungus Trichoderma reesei using the CRISPR/Cas9 system. Cell Discov. 2015, 1, 15007. [CrossRef] [PubMed] 
19. Turgeon, B.G.; Condon, B.; Liu, J.; Zhang, N. Protoplast transformation of filamentous fungi. In Molecular and Cell Biology Methods for Fungi; Sharon, A., Ed.; Humana Press: Totowa, NJ, USA, 2010; pp. 3-19.

20. Sonderegger, C.; Galgóczy, L.; Garrigues, S.; Fizil, Á.; Borics, A.; Manzanares, P.; Marx, F. A Penicillium chrysogenum-based expression system for the production of small, cysteine-rich antifungal proteins for structural and functional analyses. Microb. Cell Factories 2016, 15, 1-14. [CrossRef]

21. Latch, G.C.M.; Christensen, M.J. Artificial infection of grasses with endophytes. Ann. Appl. Biol. 1985, 107, 17-24. [CrossRef]

22. Johnson-Cicalese, J.; Secks, M.E.; Lam, C.K.; Meyer, W.A.; Murphy, J.A.; Belanger, F.C. Cross species inoculation of Chewings and strong creeping red fescues with fungal endophytes. Crop Sci. 2000, 40, 1485-1489. [CrossRef]

23. Bacon, C.W.; White, J.F., Jr. Stains, media, and procedures for analyzing endophytes. In Biotechnology of Endophytic Fungi of Grasses; Bacon, C.W., White, J.F., Jr., Eds.; CRC Press: Boca Raton, FL, USA, 1994; pp. 47-59.

24. Dellaporta, S.L.; Wood, J.; Hicks, J.B. A plant DNA miniprepartion: Version II. Plant Mol. Biol. Rep. 1983, 1, 19-21. [CrossRef]

25. Chujo, T.; Scott, B. Histone H3K9 and H3K27 methylation regulates fungal alkaloid biosynthesis in a fungal endophyte-plant symbiosis. Mol. Microbiol. 2014, 92, 413-434. [CrossRef] [PubMed]

26. Livak, K.J.; Schmittgen, T.D. Analysis of relative gene expression data using real-time quantitative PCR and the $2^{-\Delta \Delta C t}$ method. Methods 2001, 25, 402-408. [CrossRef] [PubMed]

27. Sander, J.; Joung, J. CRISPR-Cas systems for editing, regulating and targeting genomes. Nat. Biotechnol. 2014, 32, 347-355. [CrossRef] [PubMed]

28. Hassing, B.; Winter, D.; Becker, Y.; Mesarich, C.H.; Eaton, C.J.; Scott, B. Analysis of Epichloë festucae small secreted proteins in the interaction with Lolium perenne. PLoS ONE 2019, 14, e0209463. [CrossRef] [PubMed]

29. Winter, D.J.; Ganley, A.R.D.; Young, C.A.; Liachko, I.; Schardl, C.L.; Dupont, P.Y.; Berry, D.; Ram, A.; Scott, B.; Cox, M.P. Repeat elements organise 3D genome structure and mediate transcription in the filamentous fungus Epichloë festucae. PLoS Genet. 2018, 14, e1007467. [CrossRef]

30. Marraffini, L.A. The CRISPR-Cas system of Streptococcus pyogenes: Function and applications. In Streptococcus Pyogenes Basic Biology to Clinical Manifestations; Ferretti, J., Stevens, D.L.l., Fischeetti, V.A., Eds.; University of Oklahoma Health Sciences Center: Oklahoma City, TX, USA, 2016; pp. 1-13.

31. Kalderon, D.; Roberts, B.L.; Richardson, W.D.; Smith, A.E. A short amino acid sequence able to specify nuclear location. Cell 1984, 39, 499-509. [CrossRef]

32. DiCarlo, J.E.; Norville, J.E.; Mali, P.; Rios, X.; Aach, J.; Church, G.M. Genome engineering in Saccharomyces cerevisiae using CRISPR-Cas systems. Nucleic Acids Res. 2013, 41, 4336-4343. [CrossRef]

33. Matsu-ura, T.; Baek, M.; Kwon, J.; Hong, C. Efficient gene editing in Neurospora crassa with CRISPR technology. Fungal Biol. Biotechnol. 2015, 2, 4. [CrossRef]

34. Zhang, C.; Meng, X.; Wei, X.; Lu, L. Highly efficient CRISPR mutagenesis by microhomology-mediated end joining in Aspergillus fumigatus. Fungal Genet. Biol. 2016, 86, 47-57. [CrossRef]

35. Pohl, C.; Kiel, J.A.K.W.; Driessen, A.J.M.; Bovenberg, R.A.L.; Nygard, Y. CRISPR/Cas9 based genome editing of Penicillium chrysogenum. ACS Synth. Biol. 2016, 5, 754-764. [CrossRef]

36. Canzler, S.; Stadler, P.F.; Hertel, J. U6 snRNA intron insertion occurred multiple times during fungi evolution. Rna Biol. 2016, 13, 119-127. [CrossRef]

37. Arazoe, T.; Miyoshi, K.; Yamato, T.; Ogawa, T.; Ohsato, S.; Arie, T.; Kuwata, S. Tailor-made CRISPR/Cas system for highly efficient targeted gene replacement in the rice blast fungus. Biotechnol. Bioeng. 2015, 112, 2543-2549. [CrossRef] [PubMed]

38. Nødvig, C.S.; Nielsen, J.B.; Kogle, M.E.; Mortensen, U.H. A CRISPR-Cas9 system for genetic engineering of filamentous fungi. PLoS ONE 2015, 10, e0133085. [CrossRef] [PubMed]

39. Zheng, Y.M.; Lin, F.L.; Gao, H.; Zou, G.; Zhang, J.W.; Wang, G.Q.; Chen, G.D.; Zhou, Z.H.; Yao, X.S.; Hu, D. Development of a versatile and conventional technique for gene disruption in filamentous fungi based on CRISPR-Cas9 technology. Sci. Rep. 2017, 7, 9250. [CrossRef]

40. Li, J.; Zhang, Y.; Zhang, Y.; Yu, P.L.; Pan, H.; Rollins, J.A. Introduction of large sequence inserts by CRISPR-Cas9 to create pathogenicity mutants in the multinucleate filamentous pathogen Sclerotinia sclerotiorum. MBio 2019, 9, e00567-18.

41. Lukito, Y.; Lee, K.; Noorifar, N.; Green, K.A.; Winter, D.J.; Ram, A.; Hale, T.K.; Chujo, T.; Cox, M.P.; Johnson, L.J.; et al. Host infection by the grass-symbiotic fungus Epichloë festucae requires catalytically active H3K9 and H3K36 methyltransferases. bioRxiv 2020. [CrossRef]

42. Plett, J.M.; Martin, F. Reconsidering mutualistic plant-fungal interactions through the lens of effector biology. Curr. Opin. Plant Biol. 2015, 26, 45-50. [CrossRef] [PubMed]

43. Selin, C.; de Klevit, T.R.; Belmonte, M.F.; Fernando, W.G.D. Elucidating the role of effectors in plant-fungal interactions: Progress and challenges. Front. Microbiol. 2016, 7, 600. [CrossRef]

44. Uhse, S.; Djamei, A. Effectors of plant-colonizing fungi and beyond. PLoS Pathog. 2018, 14, e1006992. [CrossRef]

45. Lo Presti, L.; Lanver, D.; Schweizer, G.; Tanaka, S.; Liang, L.; Tollot, M.; Zuccaro, A.; Reissmann, S.; Kahmann, R. Fungal effectors and plant susceptibility. Annu. Rev. Plant Biol. 2015, 66, 513-545. [CrossRef] 
46. Sperschneider, J.; Gardiner, D.M.; Dodds, P.N.; Tini, F.; Covarelli, L.; Singh, K.B.; Manners, J.M.; Taylor, J.M. EffectorP: Predicting fungal effector proteins from secretomes using machine learning. New Phytol. 2016, 210, 743-761. [CrossRef] [PubMed]

47. Wang, R.; Clarke, B.B.; Belanger, F.C. Transcriptome analysis of choke stroma and asymptomatic inflorescence tissues reveals changes in gene expression in both Epichloë festucae and its host plant Festuca rubra subsp. rubra. Microorganisms $2019,7,567$. [CrossRef] [PubMed] 